\title{
Asymmetry in Orb-Webs: An Adaptation to Web Building Costs?
}

\author{
Michael Coslovsky • Samuel Zschokke
}

Revised: 26 June 2008 / Accepted: 30 July 2008 /

Published online: 23 August 2008

(C) Springer Science + Business Media, LLC 2008

\begin{abstract}
Orb-web spiders build vertically asymmetric webs, in which the lower part is larger than the upper part. One hypothesis explaining this asymmetry suggests that the spider's mass imposes higher building costs in the upper part of the web, causing the spider to reduce this part of the web. We tested this hypothesis by assessing building costs of different parts of the web. We found that the specific time-cost of building (i.e. the time required to build a certain length of silk) differed between the two parts of the web and that the difference in time-costs influenced web asymmetry. Contrary to predictions, however, building costs were larger in the lower part of the web, suggesting that additional factors affect the spider's decisions while building the web, which are likely to be prey-capture considerations.
\end{abstract}

Keywords Araneus diadematus · up-down asymmetry · gravity · optimal foraging · spider web

\section{Introduction}

Optimal Foraging Theory predicts that animals should balance the costs and benefits of foraging in an adaptive manner (Stephens and Krebs 1986). Foraging costs that are typically considered include travel time between patches or prey handling time, as

\footnotetext{
M. Coslovsky $\cdot$ S. Zschokke

Department of Environmental Sciences, Section of Conservation Biology, University of Basel, St. Johanns-Vorstadt 10, 4056 Basel, Switzerland

S. Zschokke

e-mail: samuel.zschokke@unibas.ch

M. Coslovsky $(\bowtie)$

Institute of Ecology and Evolution,

University of Bern, Baltzerstrasse 6, 3012 Bern, Switzerland

e-mail: m.coslovsky@students.unibe.ch
} 
well as search time within a patch. Reducing the different possible costs is often traded-off with the expected benefit from the caught prey (Stephens and Krebs 1986).

Spiders can be generally divided according to their foraging behavior into two ecological groups: the wandering spiders and the more sedentary web spiders (Foelix 1996). One can roughly divide the foraging behavior of the sedentary web spiders to three stages (following Vollrath 1992): (1) the spider performs patch choice when selecting a location in which to build its web; (2) the construction of the web is comparable with searching for prey within a patch; and (3) catching and retrieving prey caught on the web is comparable with central place foraging (sensu Orians and Pearson 1979). Various web spiders have developed different strategies in order to optimize their foraging behavior by developing different webs and prey catching techniques. Among the different web shapes the best known is probably the orb-web.

Orb-web spiders can be regarded central place foragers (Vollrath 1992). The theory of central place foraging assumes that the animal maximizes the energy delivery rate to a central place (Orians and Pearson 1979). In the view of orb-web spiders as central place foragers, the theory predicts that the value of a particular part of their web decreases with its distance from the central place (Orians and Pearson 1979), in their case - the hub of the web.

The building of the web is the moment in which orb-web spiders must make most decisions concerning the trade-offs between costs and benefits of their webs. It is interesting to note that these decisions occur long before any prey is encountered or noticed. Nevertheless, the spider must already in this foraging stage decide how to allocate and invest its resources and allocate the different foraging costs into the total foraging effort. These decisions, although probably based mainly on the result of past selection on the species (Vollrath 1992), may also be based on the individual spider's previous experiences (e.g. Heiling and Herberstein 1999).

The web's primary function is catching prey, with the spider itself playing an active role in the trap (Vollrath 1992; ap Rhisiart and Vollrath 1994; Zschokke et al. 2006). The web intercepts and retains insects, and informs the waiting spider about the status and the location of the prey (Vollrath 1988; Zschokke et al. 2006). The role of the spider in the trap is crucial, because capture threads of orb-webs are not strong or sticky enough to hold an insect for long (Zschokke et al. 2006), and the spider must then be fast enough to catch the prey before it escapes or tumbles away.

These facts suggest that the spider's foraging success depends both on the shape of the web, i.e. the decisions the spider takes while building the web, and on the spider's behavior once prey is encountered. These two components are likely to be related to each other.

Most vertical orb-webs show a pronounced vertical asymmetry with the lower part of the web being larger and containing more silk than the upper part (Masters and Moffat 1983; Vollrath and Mohren 1985; Herberstein and Heiling 1999). This asymmetry generally increases with size and age of the spider (Mayer 1952; Zschokke and Vollrath 1995; Heiling and Herberstein 1998; Japyassú and Adés 1998), but see (Witt and Reed 1965). Two hypotheses attempt to explain this asymmetry in view of optimal foraging theory: (1) an adaptation to web-building costs-Herberstein and Heiling (1999) found that spiders which had their mass increased built more asymmetric webs. This led them to suggest that the constraint gravity imposes on the spider is greater while building the upper part of the web than the lower part, since there, in contrast to the lower part, the spider must lift its heavy 
abdomen over its carapace. Consequently, the spider reduces the building costs by reducing the upper part of the web. The vertical asymmetry has also been explained as (2) an adaptation to prey capture-Masters and Moffat (1983), as well as ap Rhisiart and Vollrath (1994), have shown a positive relation between the spider's running speed - which is greater downward than upward - to the extent of the web in these directions. They suggested that the location of the hub equalizes the time the spider requires for catching prey in the different directions.

In this study we tested the hypothesis that the asymmetrical shape of orb-webs is an adaptation to web-building costs by videotaping spiders while they were building webs and assessing the costs of building the different parts of a web. We then compared the costs of building each part of each web to test whether they differ. We used a statistical model to examine the efficiency of the costs of building as predictors of web asymmetry.

\section{Methods}

During spring 2006 we collected 50 Araneus diadematus (Araneae, Araneidae), a common garden spider in Europe, from urban gardens in the city of Basel, Switzerland. Araneus diadematus build asymmetric webs (Vollrath and Mohren 1985; ap Rhisiart and Vollrath 1994) and the young spiders sit on the hub waiting for prey with their prosoma ("head") downward (Wirth and Barth 1992). All the spiders collected were in juvenile stages (mean $\pm \mathrm{SD}$ of spider mass on day of collection was $32.8 \pm 12.5 \mathrm{mg}$ ) estimated to be in the second or third instar by comparison to reported sizes of $A$. diadematus (Witt et al. 1972; Ramousse 1973; Vollrath et al. 1997). The spiders were kept in the laboratory (light cycle $13 \mathrm{~h}$ day/11 h night; temperature range $25-29^{\circ} \mathrm{C}$; humidity $\sim 60 \%$ ) between June and August 2006 . Each spider was kept in an individual Plexiglas frame $(30$ (height $) \times 30 \times 5 \mathrm{~cm}$ ), where it was allowed to build webs. The frames were covered from within with a net-like crack-seal tape, which provided anchoring for the webs. Frames were separated from each other by Plexiglas sheets smeared with Vaseline to prevent the spiders from attaching the webs to the sheets (for details see Zschokke and Herberstein 2005). We fed the spiders three fruit flies (Drosophila sp.) twice a week during the whole time they were kept in the laboratory. After each feeding event spiders were encouraged to build new webs by cutting holes evenly above and below the hub in the existing web. Spiders mostly built new webs after such web destruction events.

After 2 weeks of habituation to laboratory conditions we started measuring web asymmetry and building costs as described below. After 5 weeks of measurements (which made the 'before treatment' group), we allocated the spiders to two treatment groups 'weight' and 'control'. In the 'weight' group we artificially increased spider mass by gluing a small piece of metal wire on their abdomens. The added mass consisted roughly $50 \%$ of each spider's mass (following Herberstein and Heiling 1999), and was placed as close as possible to the middle of the abdomen, at its "highest" point. In the 'control' group we glued a small piece of aluminum foil each weighing roughly $1 \mathrm{mg}$ (1-5\% of spider's mass) on the spiders' abdomens.

We allocated the spiders to the groups by ranking them according to their mass before the treatment started. Each pair with the closest ranks was then split into the 
two groups by tossing a coin. This method ensured similar mean and variance of the mass for both treatment groups (mean mass \pm SD of spiders just before performing the treatment were $29.0 \pm 7.8 \mathrm{mg}$ for the 'control' group and $29.1 \pm 7.9 \mathrm{mg}$ for the 'weight' group).

The gluing process was done by briefly anaesthetizing the spiders with $\mathrm{CO}_{2}$. The glue used was a non-toxic adhesive (UHU GmbH and Co. KG, Buehl, Germany). Spiders were weighed and measured just before the treatment, and once more after the glue had dried out. After the treatment we allowed the spiders another week of habituation before commencing measurements again.

For the statistical analysis the spider's mass just before the treatment was taken as the spider's mass for the whole period before the treatment, and the spider's mass directly after the treatment was considered as the mass for measurements after the treatment. If a spider molted after the treatment it was excluded from further experiments and released at the location where it had been collected.

\section{Web Asymmetry}

We photographed the webs the spiders built before the treatment and again after the habituation period following the treatment: spiders mostly built new webs after feeding events (see above). However, not all the webs the spiders built could be used for the analysis-some webs were damaged during the photography attempt, and some photographs were not clear enough to be included in the analysis. Only spiders with at least three successful web photos were used in the analysis. From the photos we measured the vertical distances from the third outmost sticky thread, in the parts of the web above and below the hub, to the center of the hub. We shall refer to these measurements as the web's partial heights, in the parts of the web above (upper) or below (lower) the hub. We regarded the center of the hub as the mid-point between the crossings of the nearest radii above and below the hub, from the right- and left-hand sides of the hub. The two outermost sticky spiral loops often differ considerably in their mesh-size (the distance between two spirals) from the main part of the web. Due to this reason they probably play a smaller role in prey-capture since an insect hitting only one distantly spaced silk thread may often not be retained on the web, but rather tumble from the thread and escape. We therefore excluded these spirals when measuring the web's partial heights - in previous experiments (Zschokke, unpublished data) we found that excluding these threads from the analysis does not significantly alter the asymmetry measurements.

As a measure for web asymmetry, we calculated the ratio of the difference between the upper and lower parts' partial heights $\left(\mathrm{ph}_{\mathrm{u}}\right.$ and $\mathrm{ph}_{1}$ respectively) and their sum: Web Asymmetry $=\left(\mathrm{ph}_{\mathrm{u}}-\mathrm{ph}_{\mathrm{l}}\right) /\left(\mathrm{ph}_{\mathrm{u}}+\mathrm{ph}_{1}\right)$. The result gives a value between -1 to 1 , where 0 reflects an absolutely symmetric web; negative values represent webs with a larger lower part; and positive values, a web with a larger upper part. For each spider an average-web-asymmetry was calculated before and after the treatment as the mean of the web asymmetries from the different webs photographed. We used Welch's $t$-test to compare the mean web asymmetries between the treatment groups. When comparing the average web asymmetry of individual spiders before and after the treatment we used the paired $t$-test. 


\section{Web-Building Costs}

In order to assess costs of building the web we videotaped spiders while they were building webs (Sony digital video; time resolution=25 frames per second). Once we identified a spider as starting to build a web, we placed its frame in front of the camera and attempted to record the process. Some spiders did not resume the building after they had been placed in front of the camera, thus the number of videos from the different groups depended on our filming success. We succeeded in videotaping 21 occasions of web-building (14 before the treatment, five and two after it, from the 'control' and 'weight' groups respectively). The videos were then processed with a computer program (Move Watch v2.11; Benjamin and Zschokke 2002) recording the spider's position at each moment, and measuring the time and length of the spider's movements while building the web.

We divided the building costs into three components (following Zschokke 2001): (1) predation risk can be considered proportional to the time the spider requires to build the web, a time during which it is moving and may be revealed to predators. We defined the specific 'time-cost' as the time required for building the sticky spiral divided by the length of sticky silk in the upper or lower parts of the web (time-cost= sticky spiral building time/length of sticky silk); (2) motive energy - the length of movements a spider does while building its web is a rough estimate of the energy a spider spends in the process. Since gravity affects the spider in the vertical direction, moving vertically is likely to require more energy than moving horizontally. Consequently, we took into account only the vertical movement of the spider while building the web. Thus, we calculated the specific 'energy-cost' as the amount of vertical movement (measured in $\mathrm{mm}$ ) performed by the spider while building each part of the sticky spiral (upper or lower) divided by the length of sticky silk it built (energy-cost=vertical movement/length of sticky silk); and (3) material costs - (i.e. silk production) since we assumed that the costs of producing a unit length of silk do not differ between the upper and lower parts of the web, regardless of the spider's mass, we did not attempt to measure material costs.

For the analysis we calculated the 'time-' and 'energy-cost proportions'. After determining the costs (time or energy) for building the upper and the lower parts of the web, we calculated the proportion for each web built, in the same manner as we calculated the web asymmetry (see above).

We used one sample $t$-tests in order to test whether each component of the building costs differed from 0 or not. We then performed a backwards step-wise linear regression to inspect the effect various factors had on the web asymmetry.

All the statistical analysis was performed using $\mathrm{R}$ version 2.5.1 (R Development Core Team 2006). For the regression models we used the ' $\mathrm{lm}$ ' (linear model function); the ANOVA table is presented.

\section{Results}

Web Asymmetry

For calculating web asymmetry, we could use 40 spiders (out of the 50 we had collected) in the 'before treatment' group and 17 and 19 spiders in the 'control' and 
'weight' groups respectively. For comparisons before- and after-treatment, results of 12 and 14 spiders could be used from the 'control' and 'weight' groups respectively; other spiders did not have measurements both before and after the treatment:

In general, spiders had negative average web asymmetry values, indicating that almost all spiders consistently built asymmetric webs with lower parts larger than the upper parts (Fig. 1). No correlation was found between the web asymmetry and the mass of spiders from the different groups (Fig. 1; Table 1). Comparing the webs of spiders from the two treatment groups revealed no significant difference in their average asymmetries (Welch's $t$-test: $t=0.23, d f=32.52, p=0.821$; Table 1). No significant difference was detected in both groups also when comparing the average web asymmetries of webs of the same spiders before and after the treatment (paired $t$-tests, control: $t=1.37, d f=11, p=0.198$; weight: $t=1.48, d f=13, p=0.163$ ).

\section{Building Costs}

The mean costs of building the upper and the lower part of the web for each group are reported in Table 2. Since we found no difference in asymmetry of webs between the different groups, we combined the data of building costs from all groups. The mean time-cost proportion $( \pm \mathrm{SE})$ was $-0.091 \pm 0.020$, which differed significantly from 0 (one-sample $t$-test: $t=-9.91, d f=20, p<0.001$ ), indicating that the specific time-cost of building the lower part of the webs was significantly larger than that of the upper part. In other words, spiders spent more time per mm sticky-silk laid in the lower part of the web. Energy-cost proportions were significantly different from 0 as well (one-sample $t$-test: $t=7.54, d f=20, p<0.001$ ), but with a positive mean of $0.066 \pm$ 0.014 reflecting higher specific-energy-costs on average while building the upper part of the web.

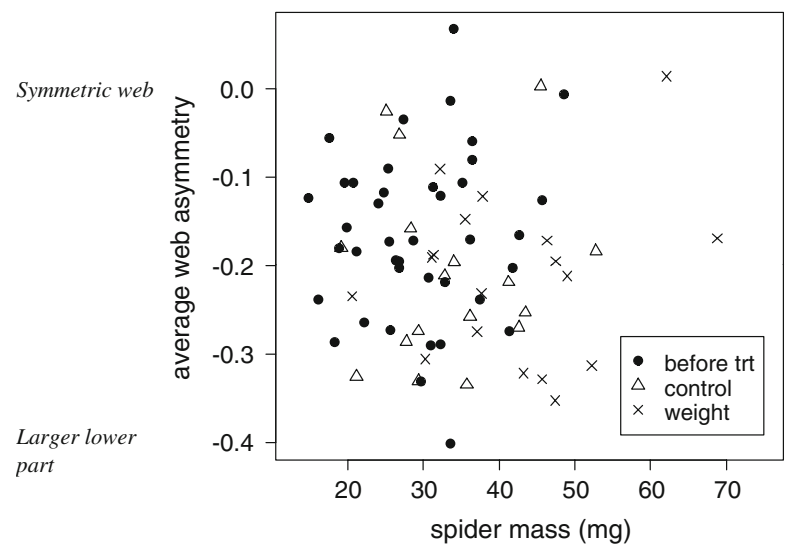

Fig. 1 Web asymmetries of spiders of three groups - a 'before treatment' group, and two treatment groups: 'control' (no additional mass) and 'weight' (metal weight of about 50\% of spider's own mass was glued on spiders) according to their mass; each point represents the average of three to five webs of the same spider. Values closer to 0 indicate higher symmetry while a negative value indicates a larger lower part of the web, and vice versa. No significant differences were found, neither between the two treatment groups, nor between the average asymmetry of each spider's webs before and after the treatment. 
Table 1 Web Asymmetry in the Different Treatment Groups

\begin{tabular}{lcccr}
\hline Treatment group & Web asymmetry (mean \pm SE) & $r$ (asymmetry-mass) & $d f$ & $p$ \\
\hline Before treatment & $-0.166 \pm 0.015$ & 0.101 & 38 & 0.536 \\
Control & $-0.209 \pm 0.025$ & 0.062 & 15 & 0.812 \\
Weight & $-0.217 \pm 0.021$ & 0.162 & 16 & 0.521 \\
\hline
\end{tabular}

Web asymmetry did not significantly differ between the groups, nor did it differ for each spider before and after the treatment. Web asymmetry was not correlated to spider mass in any of the groups

Interestingly, the smaller the difference between the time-costs of the two parts was, the more the webs were symmetrically built (Fig. 2a; Pearson's correlation, $r=-0.64$, $p=0.003)$. In contrast, no relationship was found between the energy-cost proportion and web asymmetry (Fig. 2b; Pearson's correlation, $p=0.457$ ).

To further inspect the effects of the building costs on web asymmetry, and to be able to determine which factors influence the final shape of the web and the level of asymmetry we performed a backwards stepwise-linear-regression with asymmetry of the web as the dependant variable. The variables whose effects on web asymmetry we were interested to test were included as predictor variables: group, time- and energy-cost proportions, sticky-silk length (as a measure of total web size) and second level interactions of the variables. The minimum $p$-value for exclusion was 0.2 . The final model was: asymmetry $\sim$ group+time-cost proportion, where both variables were significant (adj. $R^{2}=0.40$ ). When two possible outliers (Fig. 2a) were removed, 'time-cost proportion' remained significant but the variable 'group' was no longer significant (Table 3; adj. $R^{2}=0.46$ ).

\section{Discussion}

In our experiment, the spiders built asymmetric webs with a lower part larger than the upper one, as expected following previous studies (Masters and Moffat 1983; Vollrath and Mohren 1985; Herberstein and Heiling 1999). However, in contrast to previous results (i.e. Herberstein and Heiling 1999), webs of spiders with added mass were not more asymmetric than those of spiders with no added mass. In

Table 2 Time- and Energy-Costs (mean \pm SE) of Building the Different Parts of the Web, Above and Below the Hub, as well as the Resulting Time- and Energy-Cost Proportions in the Different Treatment Groups

\begin{tabular}{|c|c|c|c|c|c|c|c|}
\hline \multirow[t]{2}{*}{ Group } & \multicolumn{3}{|l|}{ Time-cost } & \multicolumn{3}{|l|}{ Energy-cost } & \multirow[t]{2}{*}{$N$} \\
\hline & Above hub & Below hub & Proportion & Above hub & Below hub & Proportion & \\
\hline $\begin{array}{l}\text { Before } \\
\text { treatment }\end{array}$ & $0.222 \pm 0.015$ & $0.264 \pm 0.015$ & $-0.091 \pm 0.013$ & $0.821 \pm 0.047$ & $0.731 \pm 0.037$ & $0.055 \pm 0.009$ & 14 \\
\hline Control & $0.211 \pm 0.021$ & $0.247 \pm 0.019$ & $-0.082 \pm 0.014$ & $0.783 \pm 0.081$ & $0.645 \pm 0.062$ & $0.094 \pm 0.023$ & 5 \\
\hline Weight & $0.257 \pm 0.022$ & $0.322 \pm 0.024$ & $-0.112 \pm 0.005$ & $0.859 \pm 0.115$ & $0.737 \pm 0.107$ & $0.077 \pm 0.006$ & 2 \\
\hline
\end{tabular}

Time-cost proportions were significantly smaller than 0 , implying that time-costs were larger for the lower part of the webs, whereas energy-cost proportions were significantly larger than 0 , implying that energycosts were larger while building the upper part of the webs (see text) 

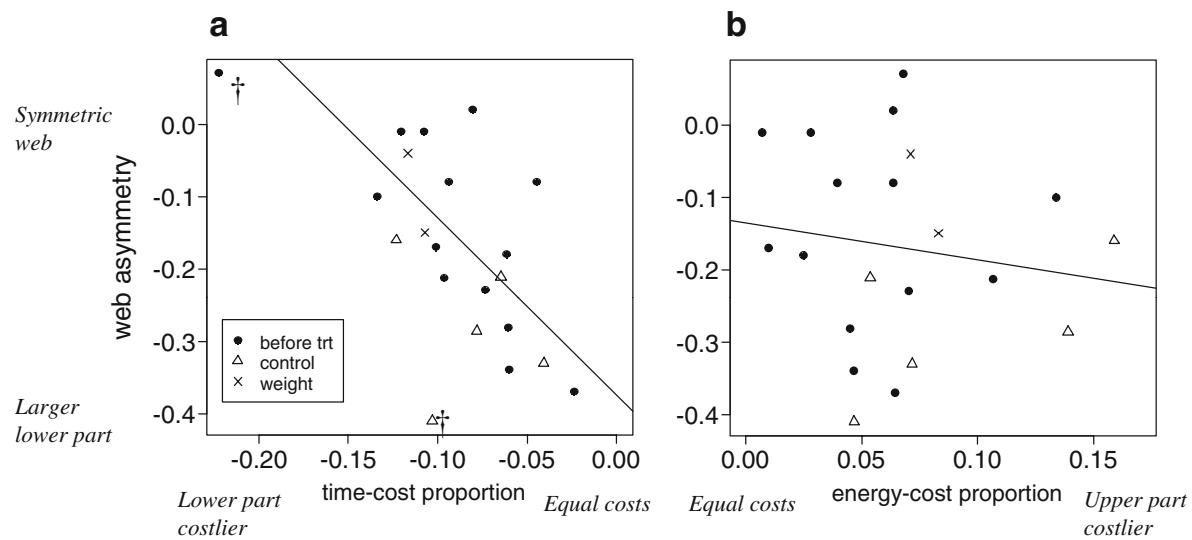

Fig. 2 a Web asymmetries vs. time-cost proportion. The specific time-costs of building the lower part of the web were higher (i.e. more time per mm of sticky silk) than in the upper part of the web. Time-cost was significantly $(p<0.003)$ correlated with the asymmetry of the web: the more it was equal between the two parts of the web, the more the web was asymmetric (points marked with daggers were considered outliers). b Web asymmetries vs. energy-cost proportion. The specific energy-costs of building the upper part of the web were larger than of the lower part. The energy-cost was not significantly correlated with the web asymmetry.

addition, we found that the specific time-costs of building webs were larger when building the lower part of the web than the upper part, and that they were a good predictor of the final asymmetry of the web.

\section{The Effect of Additional Mass on Web Asymmetry}

Herberstein and Heiling (1999) found that spiders that had their mass experimentally enlarged (either by different feeding regimes or artificially by gluing weights on their abdomens) built more asymmetric webs. In our study we could not repeat this result.

A few differences exist between the studies. For example Herberstein and Heiling (1999) used the capture area and the amount of silk invested in the different web

Table 3 ANOVA Table of Linear-Model for Web Asymmetry Resulting from Backwards-Stepwise Regression, Showing that Web Asymmetry Decreased with Increasing Time-Cost-Proportion (i.e. More Equal Time-Cost Between Building the Upper and the Lower Parts of the Web)

\begin{tabular}{llrcccc}
\hline Model & Variable & $d f$ & Sum Sq & Mean Sq & $F$ value & $p(>F)$ \\
\hline Including outliers & Group & 2 & 0.081 & 0.041 & 4.051 & $0.036^{*}$ \\
& Time-cost prop. & 1 & 0.118 & 0.118 & 11.787 & $0.003^{* *}$ \\
& Residuals & 17 & 0.170 & 0.010 & & \\
Without outliers & Group & 2 & 0.031 & 0.015 & 1.795 & 0.200 \\
& Time-cost prop. & 1 & 0.096 & 0.096 & 11.227 & $0.004^{* *}$ \\
& Residuals & 15 & 0.128 & 0.009 & & \\
\hline
\end{tabular}

Time-cost proportion remained significant after removing two outliers, whereas group was no longer significant. This reflects the importance of time-cost of building, as opposed to the other variables included in the model at the start (energy-cost proportion, sticky-silk length and second level interactions of the variables), in influencing the final shape of the web.

Asterisks represent levels of statistical significance $(*<0.05 ; * *<0.01)$ 
parts in order to calculate the web's asymmetry, and used adult spiders as well as juvenile spiders. We, on the other hand, calculated asymmetry based on the partial heights from the hub and used only juvenile spiders. Pinpointing the reason for the differing results is, thus, difficult. However, one difference between the studies that might explain the contradicting results is that Herberstein and Heiling measured the asymmetry of the first web the spiders built after the treatment, whereas we concentrated on the average asymmetry found in the spiders' webs after allowing the spiders 1 week for habituating to their new mass. This difference might explain why we could not repeat the results of Herberstein and Heiling (1999) since in their experiment the spiders, lacking web-building and prey-capture experience with their new mass, might not yet have adapted their behavior to this mass. Spiders have been shown to change their web-building behavior with experience (e.g. Heiling and Herberstein 1999). It is possible that the spiders in Herberstein and Heiling's experiment would not have been found to change their web asymmetry after time, had they been followed longer.

\section{Web-Building Costs}

The hypothesis that building costs might differ between the upper and lower parts of the web is supported by our study since we found that the building costs indeed differ between the parts of the web. We found that the required movement for building one mm of silk (i.e. energy-cost) was higher in upper part of the web, as originally predicted by Herberstein and Heiling's hypothesis (1999), but this cost of building did not significantly explain any part of the asymmetry found in the final webs. The time required for building one mm of sticky silk (i.e. time-cost) did, on the other hand, significantly explain a major part of the asymmetry of the webs. In contrast to Herberstein and Heiling's hypothesis (1999), the specific time-cost was larger in the lower part of the web. Furthermore, increasing equality in the time-cost was related to increasing web asymmetry, suggesting that the lower part of the web is more valuable to the spider, and that, whenever the building costs allow it, the spider will invest more in the lower part. This also eliminates the possibility that the relations are a result of autocorrelation, since autocorrelation would result in a positive relation between the time-cost and the web asymmetry. Due to the small sample size in the two treatment groups, we cannot draw conclusions about the role of the treatments in our regression model on web asymmetry.

Our finding that the lower part is more valuable implies that we may need to concentrate on the foraging value of the different parts of the web, and brings us back to the hypothesis that web asymmetry is an adaptation to prey-capture, as suggested by ap Rhisiart and Vollrath (1994). The higher foraging value may arise from the spiders' greater running speed downwards than upwards (Masters and Moffat 1983; ap Rhisiart and Vollrath 1994; Zschokke and Nakata, submitted). This hypothesis is also supported by preliminary results we obtained by measuring running speeds of the spiders with different mass: web asymmetry was related to the spiders' running speed upwards, although we could not find any relation between the spider's mass and its running speed or its web's asymmetry (Coslovsky 2007).

We conclude that our results support the hypothesis that the building costs differ between the upper and lower parts of orb-webs. And yet the evidence suggests that factors other than building costs also contribute to the spider's decisions on web 
asymmetry while building its web. These factors are likely to be related to preycatching decisions, as suggested by earlier studies (Masters and Moffat 1983; ap Rhisiart and Vollrath 1994).

Acknowledgments We wish to thank Dr. Daniel Bloch for his computational and statistical advice; Dr. Peter Stoll for his statistical advice and for his remarks on the manuscript; and Prof. Yael Lubin for her helpful remarks. We are also grateful to three unknown referees for their useful remarks.

\section{References}

ap Rhisiart A, Vollrath F (1994) Design features of the orb web of the spider, Araneus diadematus. Behav Ecol 5:280-287

Benjamin SP, Zschokke S (2002) A computerized method to observe spider web building behavior in a semi-natural light environment. In: Toft S, Scharff N (eds) European arachnology 2000. Aarhus University Press, Aarhus, pp 117-122

Coslovsky M (2007) Asymmetry in spider webs: an adaptation to prey capture or to web building? Master Thesis, University of Basel

Foelix RF (1996) Biology of spiders, 2nd edn. Oxford University Press, New York

Heiling AM, Herberstein ME (1998) The web of Nuctenea sclopetaria (Araneae, Araneidae): relationship between body size and web design. J Arachnol 26:91-96

Heiling AM, Herberstein ME (1999) The role of experience in web-building spiders (Araneidae). Anim Cogn 2:171-177

Herberstein ME, Heiling AM (1999) Asymmetry in spider orb webs: a result of physical constraints? Anim Behav 58:1241-1246

Japyassú HF, Adés C (1998) From complete orb to semi-orb webs: developmental transitions in the web of Nephilengys cruentata (Araneae: Tetragnathidae). Behaviour 135:931-956

Masters WM, Moffat AJM (1983) A functional explanation of top-bottom asymmetry in vertical orbwebs. Anim Behav 31:1043-1046

Mayer G (1952) Untersuchungen über Herstellung und Struktur des Radnetzes von Aranea diadema und Zilla x-notata mit besonderer Berücksichtigung des Unterschiedes von Jugend- und Altersnetzen. Z Tierpsychol 9:337-362

Orians GH, Pearson NE (1979) On the theory of central place foraging. In: Horn DJ, Stairs GR, Mitchell RD (eds) Analysis of ecological systems. Ohio State University Press, Columbus, pp 155-177

Ramousse R (1973) Body, web-building and feeding characteristics of males of the spider Araneus diadematus (Araneae: Araneidae). Psyche 80:22-47

R Development Core Team (2006) R: a language and environment for statistical computing. R Foundation for Statistical Computing, Vienna, Austria. ISBN 3-900051-07-0. Available at http://www.R-project.org

Stephens DW, Krebs JR (1986) Foraging theory. Princeton University Press, Princeton, NJ

Vollrath F (1988) Untangling the spider's web. Trends Ecol Evol 3:331-335

Vollrath F (1992) Analysis and interpretation of orb spider exploration and web-building behavior. Adv Study Behav 21:147-199

Vollrath F, Mohren W (1985) Spiral geometry in the garden spider's orb web. Naturwissenschaften 72:666-667

Vollrath F, Downes M, Krackow S (1997) Design variability in web geometry of an orb-weaving spider. Physiol Behav 62:735-743

Wirth E, Barth FG (1992) Forces in the spider orb web. J Comp Physiol A 171:359-371

Witt PN, Reed CF (1965) Spider web-building. Measurement of web geometry identifies components in a complex invertebrate behavior pattern. Science 149:1190-1197

Witt PN, Rawlings JO, Reed CF (1972) Ontogeny of web-building behavior in two orb-weaving spiders. Am Zool 12:445-454

Zschokke S (2001) Optimal orb-web construction. Adv Ethol 36:294

Zschokke S, Herberstein ME (2005) Laboratory methods for maintaining and studying web-building spiders. J Arachnol 33:205-213

Zschokke S, Vollrath F (1995) Web construction patterns in a range of orb-weaving spiders (Araneae). Eur J Entomol 92:523-541

Zschokke S, Hénaut Y, Benjamin SP, Garcia-Ballinas JA (2006) Prey-capture strategies in sympatric webbuilding spiders. Can J Zool 84:964-973 